\title{
On the circulation luminosity in uniformly rotating stars
}

\author{
H. Kähler \\ Hamburger Sternwarte, Gojenbergsweg 112, 21029 Hamburg, Germany \\ e-mail: hkaehler@hs.uni-hamburg.de \\ Received 29 November 2006 / Accepted 29 August 2007 \\ ABSTRACT

\begin{abstract}
The equations governing meridional circulation in uniformly rotating stars are discussed, allowing for turbulence and for baroclinic effects. Relations for barotropic stars with steady motions are generalized to baroclinic stars with turbulence. The circulation luminosity (i.e., the luminosity carried by circulation through a level surface) is shown to be zero in barotropic layers and positive in the bulk of a radiative envelope. The resulting effect is important in contact binaries.
\end{abstract}

Key words. stars: rotation - stars: interiors

\section{Introduction}

This paper deals with the circulation luminosity in uniformly rotating stars, i.e., the luminosity carried by circulation currents through a level surface. A theorem derived by Mestel (1965), Roxburgh et al. (1965), and Lucy (1968) states that in radiative regions the circulation luminosity vanishes. This is usually taken for granted (e.g. Eriguchi \& Müller 1991; Maeder \& Zahn 1998). But the theorem (hereafter Lucy's theorem) concernes an idealized problem since it is based on the assumptions of barotropy and steady motions. As noted by Tassoul \& Tassoul (1995), in realistic models the circulation luminosity does not vanish.

The circulation luminosity may well be unimportant in most cases. There is however an exception. A positive circulation luminosity is crucial when trying to understand the structure of contact binaries and pass sensitive observational tests such as the period-color relation (Kähler 2004, hereafter K4).

Concerning the idealized problem, for slow rotation a first-order perturbation treatment (Sweet 1950) gives a singlecell flow pattern with matter flowing outward near the pole and inward near the equator. Second-order perturbation treatments with different approximations (Gratton 1945; Öpik 1951; Maheswaran 1968) show a double-cell pattern with the sense of the circulation changing at the level surface where the density has a critical value determined by the angular velocity. The exact analytical treatment (Mestel 1966) confirms the double-cell pattern. Unfortunately, all these results suffer from deficiencies (unbalanced angular momentum, singularities at the boundaries) since viscous effects are neglected. These deficiencies reflect the fact that steady circulation currents in inviscid stars are not possible (Randers 1941) and that turbulent viscosity has to be taken into account.

To quote Tassoul (2000), large-scale mass motions in stars do not occur in isolation. On account of local instabilities they are accompanied by small-scale mass motions. A discussion of slowly rotating stars using an approximation for the eddy viscosity (Tassoul \& Tassoul 1995) shows that the classical treatment of Sweet is a reasonable approximation in the interior and that the singularities near the boundaries can be removed. The pattern in the surface layers remains unknown since it depends sensitively on uncertain parameters.
The purpose of the present paper is to discuss the equations governing meridional circulation, allowing for turbulence and for departures from barotropy, and to determine the sign of the circulation luminosity. Arguments in K4 suggest that in radiative layers the circulation luminosity is positive. Since the arguments are based on the assumption of steady motions in baroclinic layers and since large-scale motions are always accompanied by turbulence, the arguments are not conclusive. In the present paper the effects of turbulence are taken into account.

Throughout this paper we assume thermal equilibrium and uniform rotation. We use a frame of reference that rotates with the angular velocity $\boldsymbol{\Omega}$ of the figure. The discussion concerns steady motions in barotropic layers (Sect. 2), barotropic layers with turbulence (Sect. 3), and baroclinic layers (Sect. 4).

\section{Barotropic layers with steady motions}

In this section we investigate radiative layers in strict hydrostatic equilibrium, assuming steady motions. The total potential $\psi$ satisfies the equation

$\nabla^{2} \psi=4 \pi \mathrm{G} \rho-2 \Omega^{2}$,

where $\rho$ is the density, and the equation of hydrostatic equilibrium is

$\frac{1}{\rho} \nabla P=-\nabla \psi$

where $P$ is the pressure. Let $\sigma_{\psi}$ be the surface area and $\mathrm{d} \sigma_{\psi}$ a surface element of a level surface. The mean value $\langle F\rangle$ of a quantity $F$ over a level surface is defined by

$\langle F\rangle=\frac{1}{\sigma_{\psi}} \int F \mathrm{~d} \sigma_{\psi}$.

\subsection{The classical treatment}

Here Mestel's (1966) treatment will be recalled. On account of the Poincaré theorem, all thermodynamic quantities are constant on a level surface. The radiative flux can be written in the form

$\boldsymbol{F}_{\text {rad }}=f(\psi) \nabla \psi$, 
where the function $f$ defined (in the usual notation) by

$f=-\frac{4 \mathrm{ac}}{3} \frac{T^{3}}{\kappa \rho} \frac{\mathrm{d} T}{\mathrm{~d} \psi}$

is also constant on a level surface. Equations (1) and (4) imply

$\nabla \boldsymbol{F}_{\mathrm{rad}}=f\left(4 \pi \mathrm{G} \rho-2 \Omega^{2}\right)+\frac{\mathrm{d} f}{\mathrm{~d} \psi} g^{2}$,

where $g$ is the absolute value of the gravity

$\boldsymbol{g}=-\nabla \psi$.

The heat equation is

$\nabla \boldsymbol{F}_{\mathrm{rad}}=\rho \varepsilon-\rho A v \nabla \psi \quad$ with $\quad A=T \frac{\mathrm{d} s}{\mathrm{~d} \psi}$,

where $\varepsilon$ is the rate of nuclear energy generation, $s$ is the specific entropy, and $v$ is the circulation velocity. Equating the two expressions for $\nabla \boldsymbol{F}_{\text {rad }}$ we obtain

$\rho A v \nabla \psi=\rho \varepsilon-f\left(4 \pi \mathrm{G} \rho-2 \Omega^{2}\right)-\frac{\mathrm{d} f}{\mathrm{~d} \psi} g^{2}$.

Multiplying this equation by $g^{-1}$, integrating over a level surface and dividing by $\sigma_{\psi}$ we obtain

$0=\left[\rho \varepsilon-f\left(4 \pi \mathrm{G} \rho-2 \Omega^{2}\right)\right]\left\langle g^{-1}\right\rangle-\frac{\mathrm{d} f}{\mathrm{~d} \psi}\langle g\rangle$

since the net mass flow through the level surface vanishes. The last two equations give

$\rho A v \nabla \psi=\frac{\mathrm{d} f}{\mathrm{~d} \psi}\left(\frac{\langle g\rangle}{\left\langle g^{-1}\right\rangle}-g^{2}\right)$.

Use of Eq. (10) shows that the circulation inversion occurs at the level with the density $\rho_{0}$ given by

$4 \pi \mathrm{G} \rho_{0}=2 \Omega^{2}$.

Serious doubts about the validity of Eqs. (10) and (11) have been expressed by Tassoul \& Tassoul (1995) and Tassoul (2000), comparing the number of functions involved with the number of equations.

\subsection{Interpretation}

Let $m$ and $V$ denote mass and volume enclosed by a level surface, respectively, and $\bar{v}=V / m$ the mean specific volume. The local distance $\mathrm{d} n$ between neighboring level surfaces is

$\mathrm{d} n=g^{-1} \mathrm{~d} \psi$,

and neighboring level surfaces enclose the mass

$\mathrm{d} m=\left\langle g^{-1}\right\rangle \sigma_{\psi} \rho \mathrm{d} \psi$

Integrating Eq. (6) over the volume occupied by a mass shell we obtain

$\frac{\mathrm{d} l_{\mathrm{rad}}}{\mathrm{d} m}=f\left(4 \pi \mathrm{G}-\frac{2 \Omega^{2}}{\rho}\right)+\frac{1}{\rho} \frac{\mathrm{d} f}{\mathrm{~d} \psi} \frac{\langle g\rangle}{\left\langle g^{-1}\right\rangle}$.

Use of this equation shows that Eq. (10) reduces to

$\frac{\mathrm{d} l_{\mathrm{rad}}}{\mathrm{d} m}=\varepsilon$ and thus to Lucy's theorem. This confirms the doubts about the validity of Eqs. (10) and (11) in realistic models.

Since Eq. (6) is valid only in the barotropic case, we shall now derive Eq. (15) in a different way, which can be generalized to the baroclinic case. For this purpose we treat barotropic stars following Kippenhahn \& Thomas (1970). They defined quantities $\hat{u}, \hat{v}, \hat{w}$ by

$\hat{u}=\sigma_{\psi} / 4 \pi r^{2}, \quad \hat{v}=\langle g\rangle \frac{r^{2}}{\mathrm{G} m}, \quad \hat{w}=\left\langle g^{-1}\right\rangle \frac{\mathrm{G} m}{r^{2}}$,

where $r$ is the volume radius defined by

$V=4 \pi r^{3} / 3$.

We will need the product

$z:=\hat{u} \hat{v}=\frac{1}{4 \pi \mathrm{G} m} \int g \mathrm{~d} \sigma_{\psi}$.

On account of Gauss's integral theorem we have

$\int g \mathrm{~d} \sigma_{\psi}=\int_{V} \nabla^{2} \psi \mathrm{d} V$

Making use of Eq. (1) we find

$z=1-\frac{2 \Omega^{2}}{4 \pi \mathrm{G}} \bar{v}$

Multiplying this equation by $m$, taking the derivative with respect to $m$ and allowing for the relation

$\frac{\mathrm{d}}{\mathrm{d} m}(m \bar{v})=v$

(where $v=\rho^{-1}$ ) we obtain the differential equation for $z$

$m \frac{\mathrm{d} z}{\mathrm{~d} m}=1-z-\frac{2 \Omega^{2}}{4 \pi \mathrm{G} \rho}$.

The discussion of Kippenhahn \& Thomas (1970) shows that

$f=\frac{l_{\mathrm{rad}}}{4 \pi \mathrm{G} m z}$

where $l_{\text {rad }}$ is the radiative luminosity. Equations (23) and (24) give

$\frac{\mathrm{d} l_{\mathrm{rad}}}{\mathrm{d} m}=f\left(4 \pi \mathrm{G}-\frac{2 \Omega^{2}}{\rho}\right)+4 \pi \mathrm{G} m z \frac{\mathrm{d} f}{\mathrm{~d} m}$.

Since Eqs. (14) and (17) imply

$4 \pi \mathrm{G} m z \frac{\mathrm{d} f}{\mathrm{~d} m}=\frac{1}{\rho} \frac{\mathrm{d} f}{\mathrm{~d} \psi} \frac{\langle g\rangle}{\left\langle g^{-1}\right\rangle}$,

Eq. (25) reduces to Eq. (15).

\subsection{The balance of energy}

Here the balance of energy will be written in a more general form, which can be extended to turbulent layers. Since energy is transferred by radiation as well as by hydrodynamic processes, the energy flux can be written in the form

$\boldsymbol{F}=\boldsymbol{F}_{\text {rad }}+\boldsymbol{F}_{\text {hyd }}$,

where $\boldsymbol{F}_{\text {hyd }}$ denotes the flux carried by mass motions. Similarly the luminosity $l(m)$ can be split in the form

$l=l_{\mathrm{rad}}+l_{\text {hyd }}$. 
The sources $\varepsilon_{\text {hyd }}$ of the hydrodynamic luminosity are given by

$\varepsilon_{\text {hyd }}=\frac{\mathrm{d} l_{\text {hyd }}}{\mathrm{d} m}$.

The local sources $\varepsilon_{\mathrm{h}}$ of the flux carried by mass motions satify the equation

$\rho \varepsilon_{\mathrm{h}}=\nabla \boldsymbol{F}_{\text {hyd }}$.

Integrating this equation over the volume occupied by the mass shell $\mathrm{d} m$ we obtain the connection

$\varepsilon_{\mathrm{hyd}}\left\langle g^{-1}\right\rangle=\left\langle\varepsilon_{\mathrm{h}} g^{-1}\right\rangle$.

Thermal equilibrium requires

$\nabla \boldsymbol{F}_{\mathrm{rad}}+\nabla \boldsymbol{F}_{\mathrm{hyd}}=\rho \varepsilon$

and thus, making use of Eqs. (6) and (30),

$\varepsilon_{\mathrm{h}}=\varepsilon-f\left(4 \pi \mathrm{G}-\frac{2 \Omega^{2}}{\rho}\right)-\frac{1}{\rho} \frac{\mathrm{d} f}{\mathrm{~d} \psi} g^{2}$.

Equations (31) and (33) imply

$\varepsilon_{\text {hyd }}=\varepsilon-f\left(4 \pi \mathrm{G}-\frac{2 \Omega^{2}}{\rho}\right)-\frac{1}{\rho} \frac{\mathrm{d} f}{\mathrm{~d} \psi} \frac{\langle g\rangle}{\left\langle g^{-1}\right\rangle}$.

From the last two equations we obtain finally

$\varepsilon_{\mathrm{h}}=\varepsilon_{\text {hyd }}+\frac{1}{\rho} \frac{\mathrm{d} f}{\mathrm{~d} \psi}\left(\frac{\langle g\rangle}{\left\langle g^{-1}\right\rangle}-g^{2}\right)$.

\section{Barotropic layers with turbulence}

\subsection{Effects of fluctuations}

In the preceding section we assumed that all quantities under consideration are steady. Here we drop this assumption and allow for the effects of turbulence, also including convective regions. To be precise, we investigate the role of turbulence in the balance of energy, but we ignore the effects (e.g. turbulent pressure) in the equation of hydrostatic equilibrium. We ask which of the equations considered so far, if properly interpreted, remain valid. Since the potential $\psi$ is obtained on integrating the Poisson equation (1) over all mass elements, fluctuations of the potential and of the gravity can safely be neglected. Fluctuations in pressure can also be ignored since pressure equilibrium can be assumed. Equations (1) and (2) therefore remain valid if $\rho$ is taken to be the time-averaged density.

The radiative flux is a superposition of a steady flux (normal to the level surfaces) and a stochastic flux (with a vanishing time average) associated with the exchange of heat between turbulent elements. Equation (4) remains valid if $\boldsymbol{F}_{\text {rad }}$ is taken to be the steady flux and if in the definition of $f$ in Eq. (5) time averages are used. Accordingly, Eq. (6) with a time-averaged density also remains valid. Equation (8) however loses relevance since it is based on the assumption of steady motions. Therefore Eqs. (9)-(11) and Eq. (16) must also be either abandoned or modified. It is a simple matter to see that the remaining equations survive if in addition the quantities $\boldsymbol{F}_{\text {hyd }}, \varepsilon_{\mathrm{h}}, \rho \varepsilon$ are interpreted as time averages. Fluctuations of the luminosities $l_{\text {rad }}, l_{\text {hyd }}$ and of $\varepsilon_{\text {hyd }}$ are unimportant since these quantities are integrals over a level surface.

To summarize, apart from Eqs. (8)-(11) and Eq. (16) and apart from the outermost layers, the equations derived in Sect. 2 hold everywhere in a barotropic star in thermal equilibrium and uniform rotation if they are slightly modified using time averages. The Poincaré theorem must also be modified. The time averages of thermodynamic quantities are constant on level surfaces.

It remains to generalize Eqs. (8)-(11) and (16). To separate the effects of circulation (indices $\mathrm{c}$, circ) and turbulence (indices $\mathrm{t}$, turb) we write

$\varepsilon_{\mathrm{h}}=\varepsilon_{\mathrm{c}}+\varepsilon_{\mathrm{t}}, \quad \varepsilon_{\mathrm{hyd}}=\varepsilon_{\mathrm{circ}}+\varepsilon_{\mathrm{turb}}$

with the connection

$\varepsilon_{\text {circ }}\left\langle g^{-1}\right\rangle=\left\langle\varepsilon_{\mathrm{c}} g^{-1}\right\rangle$.

As shown in the Appendix, this decomposition is determined uniquely. The general discussion shows furthermore (cf. the Appendix and Eq. (100) with $\boldsymbol{b}=0$ ) that in barotropic layers (neglecting the kinetic energy)

$\rho \varepsilon_{\mathrm{c}}=\rho A v_{\mathrm{m}} \nabla \psi$,

where $\boldsymbol{v}_{\mathrm{m}}$ is the mass velocity and the remaining quantities are time averages. The condition of thermal equilibrium gives as the generalization of Eq. (8)

$\nabla \boldsymbol{F}_{\mathrm{rad}}=\rho \varepsilon-\rho A \boldsymbol{v}_{\mathrm{m}} \nabla \psi-\rho \varepsilon_{\mathrm{t}}$.

Allowing for Eq. (6) we obtain as the generalization of Eq. (9)

$\rho A v_{\mathrm{m}} \nabla \psi=\rho \varepsilon-f\left(4 \pi \mathrm{G} \rho-2 \Omega^{2}\right)-\frac{\mathrm{d} f}{\mathrm{~d} \psi} g^{2}-\rho \varepsilon_{\mathrm{t}}$.

Multiplying this equation by $g^{-1}$, integrating over a level surface and dividing by $\sigma_{\psi}$ we obtain as the generalization of Eq. (10)

$0=\left[\rho \varepsilon-f\left(4 \pi \mathrm{G} \rho-2 \Omega^{2}\right)\right]\left\langle g^{-1}\right\rangle-\frac{\mathrm{d} f}{\mathrm{~d} \psi}\langle g\rangle-\rho\left\langle\varepsilon_{\mathrm{t}} g^{-1}\right\rangle$,

where use has been made of the fact that with our choice of $\varepsilon_{\mathrm{c}}$ the small-scale motions on the time-average do not transfer mass. The last two equations give as the generalization of Eq. (11)

$\rho A v_{\mathrm{m}} \nabla \psi=\frac{\mathrm{d} f}{\mathrm{~d} \psi}\left(\frac{\langle g\rangle}{\left\langle g^{-1}\right\rangle}-g^{2}\right)+\rho \varepsilon_{\mathrm{turb}}-\rho \varepsilon_{\mathrm{t}}$.

This equation shows that results about the circulation velocity and the flow pattern depend on assumptions about the turbulence. On account of Eq. (38) the left hand side of Eq. (41) is equal to $\rho\left\langle\varepsilon_{\mathrm{c}} g^{-1}\right\rangle$, which means that

$\varepsilon_{\text {circ }}=0$.

Accordingly, in barotropic layers the circulation luminosity vanishes. In this sense Lucy's theorem is valid even in the presence of turbulence. The sources of the hydrodynamic luminosity are small-scale motions, and the generalization of Eq. (16) is

$\frac{\mathrm{d} l_{\mathrm{rad}}}{\mathrm{d} m}=\varepsilon-\varepsilon_{\mathrm{turb}}$

\subsection{Convective layers}

Convective layers in the stellar interior are in a close approximation barotropic. To this level of approximation the preceding discussion applies. In addition to small-scale convective motions there is a large-scale meridional circulation (e.g. Kippenhahn 1963). Let the circulation be neglected, i.e., let $\varepsilon_{\mathrm{c}}=0$ be assumed. Let also convective overshooting be neglected. The 
mixing-length theory can be used to determine an approximation for the (time-averaged) convective flux, which is outward directed and normal to the level surface. The Schwarzschild criterion implies that the borders of a convective region coincide with level surfaces. At the borders the convective flux vanishes. Accordingly, the convective luminosity is positive in the interior and zero at the borders. The hydrodynamic luminosity $l_{\text {hyd }}$ coincides with the convective luminosity. The derivative gives the sources or sinks $\varepsilon_{\text {hyd }}$. Usually there are sources/sinks in the inner/outer part of the convective zone. The local sources or sinks $\varepsilon_{\mathrm{h}}$ follow from Eq. (33) or from Eq. (35). In this approximation the time-averaged balance of energy is therefore fully determined.

If in a better treatment the circulation is taken into account, the local sources $\varepsilon_{\mathrm{c}}$ do not vanish, but as shown in the preceding subsection they do not contribute to the luminosity as long as departures from barotropy can be neglected. In layers with convective overshooting the hydrodynamic luminosity is negative.

As shown in the Appendix the turbulent flux is

$\boldsymbol{F}_{\text {turb }}=\left[\Delta(\rho \boldsymbol{v}) \Delta\left(h+\boldsymbol{v}^{2} / 2\right)\right]$,

where $h$ is the enthalpy, the symbol $\Delta$ denotes the deviation from the time average, and square brackets denote a time average. To see the connection with the mixing-length theory note that on account of pressure equilibrium $\Delta h=c_{\mathrm{P}} \Delta T$. Neglecting the kinetic energy we can therefore write

$F_{\text {turb }}=\rho v c_{P} \mathrm{~d} T$,

where $v$ is a characteristic velocity and $\mathrm{d} T$ is a characteristic temperature difference. Using the mixing-length theory in the usual notation (with $l_{\mathrm{m}}=$ mixing length and $h_{\mathrm{P}}=$ pressure scale height) we have

$v^{2}=g \delta\left(\nabla-\nabla_{e}\right) \frac{l_{\mathrm{m}}^{2}}{8 h_{\mathrm{P}}}, \quad \mathrm{d} T=T\left(\nabla-\nabla_{e}\right) \frac{l_{\mathrm{m}}}{2 h_{\mathrm{P}}}$,

and the expression for $F_{\text {turb }}$ in Eq. (46) coincides with the convective flux obtained in the mixing-length theory. Since

$\frac{v^{2} / 2}{c_{\mathrm{P}} \mathrm{d} T} \simeq \frac{1}{32} \ll 1$,

the neglect of the kinetic energy is justified.

\subsection{Radiative regions}

Consider next radiative regions. If fluctuations are assumed to be absent, use of Eqs. (8), (30) and (32) shows that

$\varepsilon_{\mathrm{h}}=A v \nabla \psi$.

Allowing for Eq. (11) we have

$\rho \varepsilon_{\mathrm{h}}=\frac{\mathrm{d} f}{\mathrm{~d} \psi}\left(\frac{\langle g\rangle}{\left\langle g^{-1}\right\rangle}-g^{2}\right)$.

Comparing Eqs. (35) and (50) we recover Lucy's theorem in the form

$\varepsilon_{\text {hyd }}=0$.

Unfortunately these equations are unphysical since large-scale motions require viscosity (Randers 1941) and viscosity requires small-scale motions. Therefore assumptions about the turbulence are necessary, as described in Sect. 3.1, and the assumption of barotropy is doubtful.

\section{Baroclinic layers}

Indeed a radiative envelope is baroclinic. Hydrostatic equilibrium is in conflict with radiative equilibrium. If the divergence of the radiative flux is negative/positive, matter is heated/cooled. Since the adjustment of pressure occurs on a much shorter time-scale than the adjustment of temperature, the density decreases/increases, and the resulting buoyancy forces cause upward/inward motions. Barotropic layers correspond to the idealized limiting case that infinitesimal density differences are sufficient to sustain the large-scale motions. In reality finite density differences (and thus baroclinic layers) are necessary to overcome the viscosity caused by turbulence.

\subsection{The generalized potential}

If turbulence is taken into account, the equation for hydrostatic equilibrium in stars in uniform rotation reads

$\frac{1}{\rho} \nabla P=\boldsymbol{g}=-\nabla \psi+\boldsymbol{b}$

where all quantities are time averages and $\boldsymbol{b}$ denotes the turbulent viscous force per unit volume. According to Helmholtz's decomposition theorem we can write

$\boldsymbol{b}=\boldsymbol{b}_{1}+\boldsymbol{b}_{2}$

where $\boldsymbol{b}_{1}$ is irrotational and can thus be derived from a potential $\chi$, and $\boldsymbol{b}_{2}$ is source-free, i.e.,

$\nabla \times \boldsymbol{b}_{1}=\nabla \boldsymbol{b}_{2}=0, \quad \boldsymbol{b}_{1}=-\nabla \chi$.

Following Kippenhahn (1977) we multiply Eq. (52) by a scalar function $\lambda(r, \vartheta)$ and choose this function in such a way that $\lambda \boldsymbol{g}$ can be derived from a generalized potential $\Psi$, i.e., that

$\nabla \times(\lambda \boldsymbol{g})=0, \quad \lambda \boldsymbol{g}=-\nabla \Psi$.

If this is possible we have

$\frac{\lambda}{\rho} \nabla P=-\nabla \Psi$.

This equation shows that the pressure is constant on the new equipotential surfaces, i.e., that $P=P(\Psi)$, and that $\rho / \lambda$ is also a function of $\Psi$, which means that on an equipotential surface the density varies like $\lambda$.

For a given vector field $\boldsymbol{g}$ the integrating factor $\lambda$ is a solution of the linear partial first order differential equation

$\nabla \times(\lambda \boldsymbol{g})=\lambda(\nabla \times \boldsymbol{g})+\nabla \lambda \times \boldsymbol{g}=0$,

which on account of Eqs. (52)-(54) reduces to

$\lambda\left(\nabla \times \boldsymbol{b}_{2}\right)+\nabla \lambda \times \boldsymbol{g}=0$

or equivalently to

$\nabla \times \boldsymbol{b}_{2}=-\nabla \Lambda \times \boldsymbol{g} \quad$ with $\Lambda=\ln \lambda$.

As shown by Kippenhahn any solution $\lambda$ can be used as an integrating factor. Indeed, if we take two solutions $\lambda_{1}, \lambda_{2}$ and the corresponding potentials $\Psi_{1}, \Psi_{2}$ with

$\nabla \Psi_{1}=-\lambda_{1} g, \quad \nabla \Psi_{2}=-\lambda_{2} g$

then from

$\nabla \Psi_{1} \times \nabla \Psi_{2}=\lambda_{1} \lambda_{2} \boldsymbol{g} \times \boldsymbol{g}=0$ 
we see that the equipotential surfaces of $\Psi_{1}$ and of $\Psi_{2}$ coincide. If $\lambda$ is a solution, then $c \lambda$ (where $c$ is an arbitrary constant) is also a solution. This follows from Eq. (57). If $\Lambda_{1}$ and $\Lambda_{2}$ are two solutions of Eq. (59), use of this equation shows that

$\nabla\left(\Lambda_{1}-\Lambda_{2}\right) \times g=0$,

which means that $\Lambda_{1}-\Lambda_{2}$ is constant on an equipotential surface. Accordingly, the integrating factors differ only by a constant factor. Therefore the gradients of the two potentials differ by the same factor. Imposing the condition that the potentials vanish at infinity we conclude that the two potentials also differ by the same factor.

To summarize, the integrating factor $\lambda$ and the generalized potential $\Psi$ are determined uniquely apart from the substitution

$\lambda \rightarrow c \lambda, \quad \Psi \rightarrow c \Psi$,

where $c$ is an arbitrary positive constant.

\subsection{Stellar structure equations}

In this subsection some structure equations for baroclinic stars will be derived, and a number of relations derived in Sect. 2 will be extended to baroclinic stars. Let $\sigma_{\Psi}$ be the surface area of a generalized equipotential surface, and $m$ the mass enclosed by the surface. Angle brackets now refer to these surfaces. Equation (55) shows that the local distance between neighboring surfaces is

$\mathrm{d} n=\lambda^{-1} g^{-1} \mathrm{~d} \Psi$.

Note that the distance does not depend on the choice of the integrating factor. The mass $\mathrm{d} m$ enclosed by these surfaces is

$\mathrm{d} m=\int \rho \mathrm{d} n \mathrm{~d} \sigma_{\Psi}=\mathrm{d} \Psi \int \rho \lambda^{-1} g^{-1} \mathrm{~d} \sigma_{\Psi}$

Taking into account Eq. (56) we find

$\mathrm{d} m=-\mathrm{d} P \int g^{-1} \mathrm{~d} \sigma_{\Psi}=-\sigma_{\Psi}\left\langle g^{-1}\right\rangle \mathrm{d} P$.

In a similar way we obtain for the volume $\mathrm{d} V$ enclosed by the surfaces

$\mathrm{d} V=-\sigma \Psi\left\langle\rho^{-1} g^{-1}\right\rangle \mathrm{d} P$

and for the change in the luminosity

$\mathrm{d} l=-\sigma \Psi\left\langle\varepsilon g^{-1}\right\rangle \mathrm{d} P$.

These equations imply

$\frac{\mathrm{d} V}{\mathrm{~d} m}=\frac{\left\langle\rho^{-1} g^{-1}\right\rangle}{\left\langle g^{-1}\right\rangle}, \quad \frac{\mathrm{d} l}{\mathrm{~d} m}=\frac{\left\langle\varepsilon g^{-1}\right\rangle}{\left\langle g^{-1}\right\rangle}$.

Adhering to the definitions in Eqs. (27)-(30) we obtain for the change in the hydrodynamic luminosity

$\mathrm{d} l_{\text {hyd }}=-\sigma_{\Psi}\left\langle\varepsilon_{\mathrm{h}} g^{-1}\right\rangle \mathrm{d} P$.

On the other hand we have $\mathrm{d} l_{\text {hyd }}=\varepsilon_{\text {hyd }} \mathrm{d} m$. A comparison of these two expressions shows that Eq. (31) remains valid in baroclinic stars.

We define the volume radius $r$ and the quantities $\hat{u}, \hat{v}, \hat{w}, z$ similar to those in Sect. 2.2, replacing merely the potential $\psi$ by the generalized potential $\Psi$. With these quantities Eq. (66) can be written in the form

$\frac{\mathrm{d} P}{\mathrm{~d} m}=-\frac{1}{\hat{u} \hat{w}} \frac{\mathrm{G} m}{4 \pi r^{4}}$

i.e., in the same form as in the barotropic case (Kippenhahn \& Thomas 1970), and making use of Gauss's theorem we find

$4 \pi \mathrm{G} m z=\int g \mathrm{~d} \sigma_{\Psi}=-\int \boldsymbol{g} \mathrm{d} \sigma_{\Psi}=-\int_{V} \nabla \boldsymbol{g} \mathrm{d} V$

where on account of Eq. (54)

$\nabla \boldsymbol{g}=-\nabla^{2} \psi-\nabla^{2} \chi$

The last term does not cause departures from barotropy and amounts merely to a slight change of the potential. In the remaining part of this subsection it will be ignored. Allowing for Eq. (1) we find

$z=1-\frac{2 \Omega^{2} V}{4 \pi \mathrm{G} m}$

which is the generalization of Eq. (21). Multiplying this equation by $m$ and taking the derivative with respect to $m$ we obtain the equation

$m \frac{\mathrm{d} z}{\mathrm{~d} m}=1-z-\frac{2 \Omega^{2}}{4 \pi \mathrm{G}} \frac{\mathrm{d} V}{\mathrm{~d} m}$

which generalizes Eq. (23). Next we use Eq. (24) as the definition of the function $f$. A straightforward calculation, allowing for Eq. (75), gives as the generalization of Eq. (25)

$\frac{\mathrm{d} l_{\mathrm{rad}}}{\mathrm{d} m}=f\left(4 \pi \mathrm{G}-2 \Omega^{2} \frac{\mathrm{d} V}{\mathrm{~d} m}\right)+4 \pi \mathrm{G} m z \frac{\mathrm{d} f}{\mathrm{~d} m}$.

Since $\rho / \lambda$ is constant on a surface $\Psi=$ const., a characteristic density $\tilde{\rho}(\Psi)$ can be defined by

$\tilde{\rho}=\rho / \lambda=-\mathrm{d} P / \mathrm{d} \Psi$.

With this definition we have

$4 \pi \mathrm{G} m z \frac{\mathrm{d} f}{\mathrm{~d} m}=\frac{1}{\tilde{\rho}} \frac{\mathrm{d} f}{\mathrm{~d} \psi} \frac{\langle g\rangle}{\left\langle g^{-1}\right\rangle}$,

and Eq. (76) can be written in the form

$\frac{\mathrm{d} l_{\mathrm{rad}}}{\mathrm{d} m}=f\left(4 \pi \mathrm{G}-2 \Omega^{2} \frac{\mathrm{d} V}{\mathrm{~d} m}\right)+\frac{1}{\tilde{\rho}} \frac{\mathrm{d} f}{\mathrm{~d} \Psi} \frac{\langle g\rangle}{\left\langle g^{-1}\right\rangle}$

which is the generalization of Eq. (15). From the condition

$\frac{\mathrm{d} l_{\mathrm{rad}}}{\mathrm{d} m}+\varepsilon_{\mathrm{hyd}}=\frac{\mathrm{d} l}{\mathrm{~d} m}$

we obtain the generalization of Eq. (34) and thus of Eq. (10)

$\varepsilon_{\text {hyd }}=\frac{\left\langle\varepsilon g^{-1}\right\rangle}{\left\langle g^{-1}\right\rangle}-f\left(4 \pi \mathrm{G}-2 \Omega^{2} \frac{\mathrm{d} V}{\mathrm{~d} m}\right)-\frac{1}{\tilde{\rho}} \frac{\mathrm{d} f}{\mathrm{~d} \Psi} \frac{\langle g\rangle}{\left\langle g^{-1}\right\rangle}$ 


\subsection{The circulation luminosity}

As shown in the appendix, the energy flux carried by circulation currents is

$\boldsymbol{F}_{\text {circ }}=\rho \boldsymbol{v}_{\mathrm{m}}\left(h+\psi+\boldsymbol{v}^{2} / 2\right)$,

where all quantities are time averages. Since $\nabla\left(\rho \boldsymbol{v}_{\mathrm{m}}\right)=0$ the sources and sinks are

$\rho \varepsilon_{\mathrm{c}}=\nabla \boldsymbol{F}_{\text {circ }}=\rho \boldsymbol{v}_{\mathrm{m}} \nabla\left(h+\psi+\boldsymbol{v}^{2} / 2\right)$.

The circulation luminosity $l_{\text {circ }}$ carried through a surface $\psi=$ const. is

$l_{\text {circ }}=\int \boldsymbol{F}_{\text {circ }} \mathrm{d} \sigma_{\psi}$.

Since the kinetic energy can be neglected and since the net mass flux vanishes we find

$l_{\text {circ }}=\int \rho \boldsymbol{v}_{\mathrm{m}}(h-\bar{h}) \mathrm{d} \sigma_{\psi}$,

where $\bar{h}$ is the mean enthalpy on the level surface.

We now apply this equation to a radiative envelope. Recall that the envelope is divided into cells. In the barotropic case there is in each hemisphere an inner cell (where matter is rising around the poles and falling around the equator) as well as an outer cell. When viscous effects are taken into account, the inner cell persists, as shown by Tassoul \& Tassoul (1995), but nothing can be said on outer cells. In the following discussion we confine ourselves to the inner cell.

If on a surface $\psi=$ const. the enthalpy (or equivalently the temperature) is larger/smaller around the poles than around the equator, the integrand in Eq. (85) is positive/negative almost everywhere, and the circulation luminosity is positive/negative. This result can be written in the form

$l_{\text {circ }}\left(e_{\psi}-e_{T}\right)>0$,

where $e_{\psi}$ and $e_{T}$ denote the excentricity of the surfaces of constant potential and constant temperature, respectively. A corresponding notation will be used for the excentricities of isobaric, isopycnic and isentropic surfaces. Taking the curl of Eq. (52) we obtain

$\nabla \frac{1}{\rho} \times \nabla P=\nabla \times \boldsymbol{b}_{2}$

Making use of Gauss's theorem, from Eqs. (54) we find

$$
\int \boldsymbol{b}_{2} \mathrm{~d} \sigma_{\psi}=0
$$

Accordingly, on a level surface the product $\boldsymbol{g} \boldsymbol{b}_{2}$ changes sign. Since viscous forces tend to resist the large-scale motion, $\boldsymbol{g} \boldsymbol{b}_{2}$ is positive around the poles and negative around the equator. Use of Eq. (87) shows that $e_{\rho}>e_{P}$ and thus also

$e_{T}<e_{P}<e_{\rho}<e_{s}$

It remains to include $e_{\psi}$. When following the mean motion in the northern hemisphere, in the outer/inner part of the cell the colatitude $\vartheta$ increases/decreases. Since we can safely assume that $\boldsymbol{v}_{\mathrm{m}} \boldsymbol{b}$ is negative we see from Eq. (52) that $e_{\psi}<e_{P}$ in the inner part and

$e_{\psi}>e_{P}$ in the outer part, which covers the bulk of the cell. Without further arguments nothing more can be said on the inner part. In the outer part however Eqs. (86), (89) and (90) imply

$l_{\text {circ }}>0$.

Accordingly, in the bulk of a radiative envelope the circulation luminosity is positive. The internal mass motions provide a reduction of the radiative luminosity and thus of the temperature gradient. This is as in convective regions apart from layers with overshooting.

Energy considerations allow an interpretation. Let $l_{\mathrm{c}}$ denote the core luminosity generated by nuclear reactions in a rotating main sequence star. Thermal equilibrium requires

$l_{\mathrm{c}}=4 \pi R^{2} \sigma T_{\mathrm{eff}}^{4}$,

where $R$ and $T_{\text {eff }}$ are mean values of radius and effective temperature, respectively. We shall use this equation to ask for the effects of a decrease in the temperature gradient $\nabla$. When doing so, we may assume that the core luminosity depends on the central temperature $T_{\mathrm{c}}$ only. In a general discussion $l_{\mathrm{c}}$ is proportional to powers of $T_{\mathrm{c}}$ and other quantities, e.g., central density. In the present discussion these other effects can be taken into account modifying the power of $T_{\mathrm{c}}$. We therefore have

$4 \pi R^{2} \sigma=l_{\mathrm{c}}\left(T_{\mathrm{c}}\right) T_{\mathrm{eff}}^{-4} \quad$ with $\quad \mathrm{d} l_{\mathrm{c}} / \mathrm{d} T_{\mathrm{c}}>0$.

A decrease in $T_{\mathrm{c}}$ (for fixed $T_{\text {eff }}$ ) or an increase of $T_{\text {eff }}$ (for fixed $T_{\mathrm{c}}$ ) causes a decrease of $R$. This result strongly suggests that a decrease of the temperature gradient is accompanied by a contraction of the star.

This has been confirmed investigating barotropic models of rotating main sequence stars. Starting with a model with a vanishing circulation luminosity and passing over to a model with a positive circulation luminosity we found that in each mass shell the polar as well as the equatorial radius decreases. This contraction causes a decrease of the gravitational energy and thus, by virtue of the virial theorem, a decrease of the total energy of the star. Apparently the star settles down in the state of lowest energy rendered possible by internal mass motions.

In order to express the circulation luminosity in terms of a characteristic circulation velocity $v_{\text {circ }}$ and temperature difference $(\Delta T)_{\text {circ }}$ we write Eq. (85) in the form

$l_{\text {circ }}=c_{P} \int \rho \boldsymbol{v}_{\mathrm{m}} \boldsymbol{n}(T-\bar{T}) \mathrm{d} \sigma_{\psi}$,

where $\boldsymbol{n}$ is the unit normal vector on the level surface. The circulation luminosity is caused by a correlation of the quantities $\rho \boldsymbol{v}_{\mathrm{m}} \boldsymbol{n}$ and $T-\bar{T}$. These quantities are positive at the poles and negative at the equator. On the level surface under consideration they depend only on $\mu=\cos \vartheta$, for symmetry reasons they are even functions of $\mu$, and they vanish upon averaging. A reasonable approximation is therefore

$\rho \boldsymbol{v}_{\mathrm{m}} \boldsymbol{n}=a P_{2}(\mu), \quad T-\bar{T}=b P_{2}(\mu)$

where $P_{2}$ is a Legendre polynomial and $a, b$ are positive constants. Approximating the level surface by a sphere with

$\mathrm{d} \sigma_{\psi}=2 \pi r^{2} \sin \vartheta \mathrm{d} \vartheta=-2 \pi r^{2} \mathrm{~d} \mu$

we obtain

$l_{\text {circ }} \approx-2 \pi r^{2} c_{P} a b \int_{1}^{-1} P_{2}(\mu)^{2} \mathrm{~d} \mu=\frac{4}{5} \pi r^{2} c_{P} a b$. 
Substituting $a \rightarrow \rho v_{\text {circ }}, b \rightarrow(\Delta T)_{\text {circ }}$ we have

$l_{\text {circ }} \approx \frac{4}{5} \pi r^{2} \rho v_{\text {circ }} c_{P}(\Delta T)_{\text {circ }}$.

The averaged flux carried by circulation through the level surface is therefore

$F_{\text {circ }} \approx \frac{1}{5} \rho v_{\text {circ }} c_{P}(\Delta T)_{\text {circ }}$.

\subsection{Sources and sinks}

Since the boundary of a cell is formed of streamlines and since the flux $\boldsymbol{F}_{\text {circ }}$ and the mass velocity $\boldsymbol{v}_{\mathrm{m}}$ have the same direction, the effect of circulation is a redistribution of energy within the cell.

As shown in the previous subsection, in the bulk of the inner cell the circulation luminosity is positive. There are therefore sources $\left(\varepsilon_{\text {circ }}>0\right)$ in the interior and sinks $\left(\varepsilon_{\text {circ }}<0\right)$ in the outer layers of the cell. To find these sources and sinks, we start from the local sources or sinks of the flux $\boldsymbol{F}_{\text {circ }}$ as given in Eq. (83). Neglecting the kinetic energy and using Eqs. (52) and the thermodynamic relation $\nabla h=T \nabla s+\rho^{-1} \nabla P$ we obtain

$\rho \varepsilon_{\mathrm{c}}=\rho T \boldsymbol{v}_{\mathrm{m}} \nabla s+\rho \boldsymbol{v}_{\mathrm{m}} \boldsymbol{b}$.

The first term on the RHS represents energy gains or losses in the form of heat. The second term represents the work done against the viscous forces. The sources or sinks of the circulation luminosity follow on integrating $\rho \varepsilon_{\mathrm{c}}$ over the volume occupied by a mass shell.

The first term on the RHS of Eq. (100) gives a positive contribution to $\varepsilon_{\text {circ }}$. This can be seen on integrating $\rho \varepsilon_{\mathrm{c}}$ over the volume enclosed by two neigboring surfaces of constant entropy, making use of the inequality $e_{T}<e_{s}$ (see K4). The second term is negative. The contribution to $\varepsilon_{\text {circ }}$ is therefore also negative. Since the circulation luminosity is positive, in the inner/outer part of the cell the first/second contribution is dominating.

Combining Eq. (100) with Eqs. (30), (32), and (36) we can write the equation of energy conservation in the form

$\rho T \boldsymbol{v}_{\mathrm{m}} \nabla s+\nabla \boldsymbol{F}_{\text {rad }}+\nabla \boldsymbol{F}_{\text {turb }}+\rho \boldsymbol{v}_{\mathrm{m}} \boldsymbol{b}=\rho \boldsymbol{\varepsilon}$.

In the usual treatment of circulation the last term on the LHS is missing. This is justified only if departures from barotropy can be neglected.

\subsection{Application to contact binaries}

Contact binaries show that the effects of the circulation luminosity can be important. Recall that the two components have similar surface temperatures. This requires an energy flow from the primary to the secondary in a common turbulent envelope. If in a contact binary model the circulation in the interior of the components is neglected, the condition of similar surface temperatures is in conflict with Roche geometry since the secondary's radius is too large. The solution of this conflict requires a contraction of the secondary and thus (as discussed in Sect. 1 of K4) a decrease in the temperature gradient caused by a positive circulation luminosity. The effects of a similar circulation luminosity in the primary are unimportant.

The resulting treatment of contact binaries in K4 passes the observational tests, including the period-color relation, which is a very sensitive test. It requires a circulation luminosity in the secondary's interior that is comparable with the radiative luminosity. As a typical example consider the system AB And with

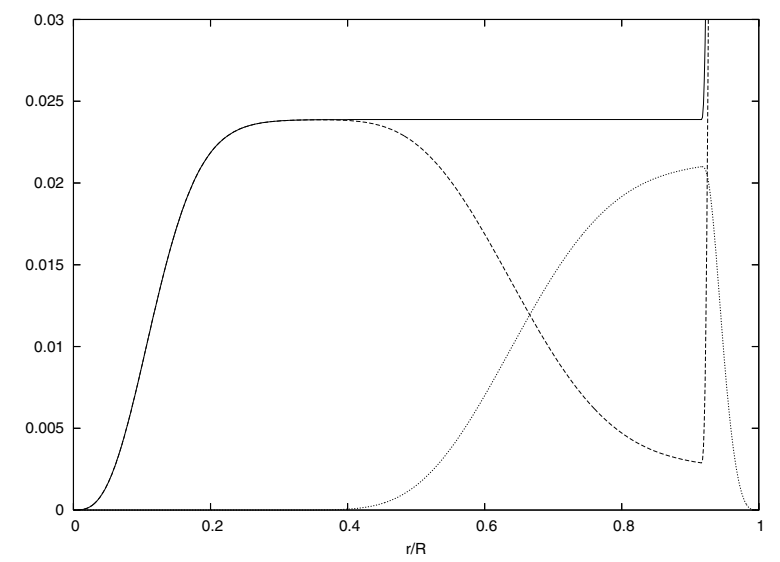

Fig. 1. Luminosities (in solar units) in the secondary of the contact system $\mathrm{AB}$ And as a function of the fractional radius (see text).

the mass $M=1.5 M_{\odot}$, the mass ratio $q=0.491$, and the period $P=0.332$ day. The geometry of the system and the variation of the gravity on a level surface in the secondary are shown in Figs. 25 and 28 in K4. The primary is evolved, but the secondary is unevolved. Figure 1 shows luminosities in the secondary as a function of the fractional radius. The core luminosity is small $\left(0.024 L_{\odot}\right)$. In the common envelope a much larger luminosity (about $0.6 L_{\odot}$ ) is transferred from the primary. The resulting increase in luminosity escapes notice in the diagram. In radiative layers $(r / R \leq 0.872)$ the total luminosity (solid line) is the sum of the radiative luminosity (dashed line) and the circulation luminosity (dotted line). In a large part of the interior the circulation luminosity is comparable with (or even larger than) the radiative luminosity. This result is reliable although details are uncertain since they depend on the choice of the circulation function (cf. Fig. 8 in K4).

Is the result also reasonable from the viewpoint of circulation velocities and temperature differences on level surfaces? Recall that convection in the stellar interior is a very effective process, with typical velocities $v=1 \ldots 100 \mathrm{~m} \mathrm{~s}^{-1}$ and temperature differences $\mathrm{d} T=10^{-2} \ldots 1 \mathrm{~K}$. Note that, for a given velocity and temperature difference, the average flux carried by circulation (Eq. (99)) differs from the convective flux (Eq. (46)) only by the factor $1 / 5$ (this geometrical factor arises since the circulation velocity, in contrast to the convective velocity, is not usually perpendicular to the level surface). Accordingly, if the product of velocity and temperature difference is comparable, circulation is only slightly less effective than convection.

As an example consider the top of the radiative interior of the secondary of $\mathrm{AB}$ And with $l_{\text {circ }}=0.02 L_{\odot}, r=0.702 R_{\odot}$, $\rho=0.014 \mathrm{~g} \mathrm{~cm}^{-3}$, and mean molecular weight $\mu=0.6165$. From Eq. (98) we obtain

$v_{\text {circ }}(\Delta T)_{\text {circ }} \approx 27 \mathrm{~m} \mathrm{~s}^{-1} \mathrm{~K}$.

Thus a velocity $v_{\text {circ }}=1 \mathrm{~m} \mathrm{~s}^{-1}$ would be accompanied by a temperature difference of $27 \mathrm{~K}$, which is very small compared with the mean temperature of $4 \times 10^{5} \mathrm{~K}$. An example for deeper layers is the level surface with $r / R=0.7$ and $l_{\text {circ }}=0.015 L_{\odot}$. Assuming again the velocity $v_{\text {circ }}=1 \mathrm{~m} \mathrm{~s}^{-1}$ we obtain a temperature difference of $2.5 \mathrm{~K}$.

These examples show that velocities of few $\mathrm{m} \mathrm{s}^{-1}$ and temperature differences of few $\mathrm{K}$ are sufficient to give a circulation luminosity comparable with the radiative luminosity. Separate estimates of velocity or temperature difference are presumably not possible in the absence of a proper hydrodynamic treatment 
of contact binaries, but the velocity is certain to be much larger than in single stars.

\section{Concluding remarks}

This paper discusses the equations governing meridional circulation and the sign of the circulation luminosity. The rotation was assumed to be uniform. Although in many real stars other rotation laws may be more realistic, e.g., shellular rotation laws as proposed by Maeder \& Zahn (1998), the assumption of uniform rotation is justified in a theoretical discussion and certainly realistic when studying the components of close binaries with strong tidal synchronisation.

The discussion is severely limited by the lack of a reliable treatment of turbulence. In particular, according to Zahn (1992) the horizontal turbulence is much more important than the turbulence in the vertical direction. This horizontal turbulence carries some flux that affects the vertical flux of the circulation, possibly in a significant way. Nevertheless, we have been able to show that in the bulk of a radiative envelope the circulation luminosity is positive.

The resulting effects are presumably small in single stars but large in contact binaries. In particular, in contact binaries the circulation velocity is much larger than in single stars. Concerning this point, recall that in single stars circulation is driven by the conflict between strict hydrostatic equilibrium (absence of internal mass motions) and radiative equilibrium. In contact binaries this conflict is larger since the rotation is fast and the variation of the effective gravity on a level surface is also large. Much more violent, however, is the conflict between Roche geometry and the condition of similar surface temperatures in the two components, which is also a condition for approximate hydrostatic equilibrium in the common turbulent envelope.

In the treatment of single stars it is almost always assumed that the circulation luminosity vanishes. It remains to be investigated whether this simplifying assumption is justified as an approximation.

\section{Appendix A: The hydrodynamic flux}

Here we derive an expression for the hydrodynamic flux in turbulent layers. As noted in Sect. 3.1 fluctuations of the potential $\psi$ can be neglected. Other quantities depend on time. Time averages will be denoted by square brackets. The equation of continuity reads

$\frac{\partial \rho}{\partial t}=-\nabla(\rho \boldsymbol{v})$

Since molecular and radiative viscosity can be neglected, the momentum equation is

$\frac{\mathrm{d} v}{\mathrm{~d} t}+2 \Omega \times \boldsymbol{v}=-\nabla \psi-\frac{1}{\rho} \nabla P$

where

$\frac{\mathrm{d} v}{\mathrm{~d} t}=\frac{\partial \boldsymbol{v}}{\mathrm{d} t}+(\boldsymbol{v} \nabla) \boldsymbol{v}$

Making use of the identity

$(\boldsymbol{v} \nabla) \boldsymbol{v}=\nabla\left(\frac{\boldsymbol{v}^{2}}{2}\right)+(\nabla \times \boldsymbol{v}) \times \boldsymbol{v}$ we find

$\frac{\partial \boldsymbol{v}}{\mathrm{d} t}+\nabla\left(\frac{\boldsymbol{v}^{2}}{2}\right)+(2 \Omega+\nabla \times \boldsymbol{v}) \times \boldsymbol{v}=-\nabla \psi-\frac{1}{\rho} \nabla P$.

The heat equation (i.e. the first law in a co-moving frame) reads

$\nabla \boldsymbol{F}_{\mathrm{rad}}=\rho \varepsilon-\rho \frac{\mathrm{d} s}{\mathrm{~d} t}$.

Using the thermodynamic relation $T \mathrm{~d} s=\mathrm{d} h-\rho^{-1} \mathrm{~d} P$ (where $h$ is the specific enthalpy) we obtain

$\nabla \boldsymbol{F}_{\mathrm{rad}}=\rho \varepsilon-\rho \boldsymbol{v} \nabla h-\rho \frac{\partial h}{\partial t}+\boldsymbol{v} \nabla P+\frac{\partial P}{\partial t}$.

Next we use the dot product of Eq. (A.5) with $\rho \boldsymbol{v}$ to eliminate $v \nabla P$. The result is

$\nabla \boldsymbol{F}_{\mathrm{rad}}=\rho \varepsilon-\rho \boldsymbol{v} \nabla X-\rho \frac{\partial h}{\partial t}+\frac{\partial P}{\partial t}$

with the abbreviation $X=h+\psi+v^{2} / 2$. On account of the equation of continuity we have

$\rho \boldsymbol{v} \nabla X=\nabla(\rho \boldsymbol{v} X)+X \frac{\partial \rho}{\partial t}$

Using this equation and recalling that $\partial \psi / \partial t=0$ we find

$\nabla \boldsymbol{F}_{\mathrm{rad}}+\nabla \boldsymbol{F}+\frac{\partial}{\partial t}(\rho X)-\frac{\partial P}{\partial t}=\rho \varepsilon$

where $\boldsymbol{F}=\rho \boldsymbol{v} X$. The time average of this equation is

$\left[\nabla \boldsymbol{F}_{\mathrm{rad}}\right]+[\nabla \boldsymbol{F}]=[\rho \varepsilon]$,

while the equation of thermal equilibrium reads

$\left[\nabla \boldsymbol{F}_{\text {rad }}\right]+\left[\nabla \boldsymbol{F}_{\text {hyd }}\right]=[\rho \varepsilon]$.

A comparison shows that

$\left[\boldsymbol{F}_{\text {hyd }}\right]=\left[\rho \boldsymbol{v}\left(h+\psi+\boldsymbol{v}^{2} / 2\right)\right]$.

Using the symbol $\Delta$ to denote deviations from the time average we have

$h=[h]+\Delta h, \quad \boldsymbol{v}^{2}=\left[\boldsymbol{v}^{2}\right]+\Delta\left(\boldsymbol{v}^{2}\right)$

and

$\rho \boldsymbol{v}=[\rho] \boldsymbol{v}_{\mathrm{m}}+\Delta(\rho \boldsymbol{v})$,

where $\boldsymbol{v}_{\mathrm{m}}$ is the mass velocity defined by

$[\rho] \boldsymbol{v}_{\mathrm{m}}=[\rho \boldsymbol{v}]$.

Inserting these expressions into Eq. (A.13) we find that

$\left[\boldsymbol{F}_{\text {hyd }}\right]=\boldsymbol{F}_{\text {circ }}+\boldsymbol{F}_{\text {turb }}$

with

$\boldsymbol{F}_{\text {circ }}=[\rho] \boldsymbol{v}_{\mathrm{m}}\left(\left[h+\boldsymbol{v}^{2} / 2\right]+\psi\right)$

and

$\boldsymbol{F}_{\text {turb }}=\left[\Delta(\rho \boldsymbol{v}) \Delta\left(h+\boldsymbol{v}^{2} / 2\right)\right]$,

i.e., that the hydrodynamic flux can be split into contributions from circulation and from turbulence. 


\section{References}

Eddington, A. S. 1925, The Observatory, 48, 73 Eriguchi, Y., \& Müller, E. 1991, A\&A, 248, 435

Gratton, L. 1945, Mem. Soc. Astron. It., 17, 5

Kähler, H. 2004, A\&A, 414, 317

Kippenhahn, R. 1963, ApJ, 137, 664

Kippenhahn, R. 1977, A\&A, 58, 267

Kippenhahn, R., \& Thomas H.-C. 1970, in Stellar Rotation, ed. A. Slettebak (Dordrecht: Reidel)

Lucy, L. B. 1968, ApJ, 151, 1123

Maeder, A., \& Zahn, J.-P. 1998, A\&A, 334,1000

Maheswaran, M. 1968, MNRAS, 140, 93
Mestel, L. 1965, in Stars and Stellar Systems, Vol. VIII (Chicago, London: The University of Chicago Press)

Mestel, L. 1966, ZAp, 63, 196

Öpik, E. J. 1951, MNRAS, 111, 278

Randers, G. 1941, ApJ, 94, 109

Roxburgh, I. W., Griffith, J. S., \& Sweet, P. A. 1965, ZAp 61, 203

Sweet, P. A. 1950, MNRAS, 110, 548

Tassoul, J.-L. 2000, Stellar rotation (Cambridge: Cambridge Univ. Press)

Tassoul, M., \& Tassoul, J. L. 1995, ApJ, 440, 789

Vogt, H., 1925, Astron. Nachr. 223, 229

von Zeipel, H. 1924, MNRAS, 84, 665

Zahn, J.-P. 1992, A\&A, 265, 115 